\title{
Another twist in the histone memory code
}

\author{
Cell Research (2015) 25:151-152. doi:10.1038/cr.2014.134; published online 24 October 2014
}

\begin{abstract}
Transcription is a highly regulated process and several studies have examined the role of transcription factors and various epigenetic regulators in memory formation. In a recent paper in Nature, Zokvic and colleagues show an important role for a novel regulator of chromatin structure (the incorporation of histone variants) in memory formation.
\end{abstract}

It has been known since the 1960s that the formation of long-term memory critically depends on transcription. For example, Agranoff and colleagues showed that the RNA synthesis inhibitor actinomycin D impaired memory formation in goldfish if administered immediately after training, but had no effect on memory if administered $1 \mathrm{~h}$ after training [1]. Transcription is highly regulated and involves a coordinated response of several players and processes, including transcription factors (regulatory proteins which activate or inhibit the transcription of DNA by binding to specific DNA sequences) and chromatin remodeling (which allows the access of transcription factors to the DNA). Important roles for transcription factors (including CREB, MEF2 and others) [24] and various chromatin modifications $[5,6]$ in memory formation have been previously described.

Roughly 2 meters of DNA is packed into the nucleus of all eukaryotic cells. Cells are able to house this vast quantity of DNA because the negativelycharged DNA is tightly wound around positively-charged histone proteins. Histone-wrapped DNA (chromatin) is relatively inaccessible to the transcription machinery. However, parts of the DNA can "unwind" thereby allowing access to transcription factors. The conformational state of the DNA is also mediated by several processes. For instance, histones may be post-transcriptionally modified (e.g., phosphorylation, methylation, acetylation, sumoylation and ubiquitination [7]). The combinations of these histone "marks" (sometimes referred to as the "histone code") determine which transcription factors can bind to specific DNA regions to regulate the expression of particular genes. Chromatin architecture may also be modulated by the incorporation of histone variants [8]. The core histones come in 4 flavors (H2A, H2B, H3 and $\mathrm{H} 4)$. Histone variants are non-allelic forms of the conventional core histones which are expressed at comparatively lower levels. All conventional histones, except $\mathrm{H} 4$, have histone variants. One of the most highly conserved histone variant is $\mathrm{H} 2 \mathrm{~A} . \mathrm{Z}$ (which may replace $\mathrm{H} 2 \mathrm{~A}$ ).

Although great strides have been made in understanding the transcriptional processes mediating memory formation, the present paper by Zovkic and colleagues [9] represents the first to examine a potential role for the incorporation of histone variants in memory formation. This is remarkable because little is known about the function of histone variants in general, and even less is known about the role of histone variant replacement in memory. To examine long-term memory formation in mice, Zovkic and colleagues used Pavlovian fear/threat conditioning in which mice learn to associate a particular place (context) with an aversive footshock. When subsequently placed back in the context (1 day or even 30 days later), mice show a defensive response (freez- ing behavior). This type of context memory initially depends on the dorsal hippocampus, but over time, this memory trace is thought to re-organize in the brain, such that it becomes increasingly dependent on the cortex, including in particular the medial prefrontal cortex (mPFC) [10]. This process (referred to as systems consolidation) is thought to be mediated by time-limited interactions between the hippocampus and cortex. Contextual memories are thought to be rapidly encoded in the hippocampus whereas this knowledge if more slowly is encoded in cortical circuits.

The current paper examined the role of the histone variant H2A.Z during memory consolidation over this same extended time course. First, Zokvic and colleagues found that H2A.Z levels are decreased in the CA1 region of the dorsal hippocampus in the minutes, but not hours, following training (consistent with the notion that H2A.Z plays a time-limited role in initial memory consolidation). This posttraining decrease in H2A.Z level was accompanied by a decrease in H2A.Z binding to key transcriptional start sites of several "memory promoter" genes (e.g., Npas4, Arc, Fos, Egr1), but an increase in incorporation of H2A.Z into a "memory repressor" gene $(P p p 3 c a)$. Together, these data suggest that H2A.Z in the dorsal hippocampus may serve to negatively regulate memory formation in the minutes following training. To test this idea, this group examined the effects of locally decreasing H2A.Z levels using viral vectors to express a construct that reduces levels of H2A.Z mRNA and protein. Microinjecting this inhibitory construct into the dorsal hip- 
pocampus of mice prior to fear training increased fear memory (freezing) measured 1 day after training, suggesting that H2A.Z normally restricts memory formation. The memory enhancement was also observed 30 days following training, suggesting that relieving the inhibitory brake of H2A.Z in the dorsal hippocampus enhances initial memory consolidation and that this strengthened hippocampal trace also instructs the cortex. However, what about the role of H2A.Z in the cortex?

To examine this, Zovkic and colleagues microinjected their H2A.Z antagonist into $\mathrm{mPFC}$ prior to fear conditioning. Memory tested either 7 or 30 days after training was enhanced. However, memory was unaffected if tested 1 day after training. This finding suggests that in the mPFC H2A.Z is not important for the expression of the initial memory trace, but that H2A.Z normally restrains the mPFC component of the memory trace that gradually emerges following training. These results agree with the general notion that cortical memory circuits integrate information more slowly than the hippocampus. Furthermore, they suggest that similar processes are engaged at different times in these different brain regions, reflecting, perhaps, evolution's penchant for re-purposing mechanisms.

Memory formation and storage involves an orchestrated response of finely-tuned processes across several brain regions. By showing a unique, brain region-dependent and timelimited role for histone variants in the process, the current study adds yet another layer of complexity (a new twist in the histone code) to this process. We stand in awe of this complexity and are reminded of that sentiment forwarded by a pioneering memory researcher. To paraphrase Karl S Lashely [11], we sometimes feel, in reviewing the evidence, that the necessary conclusion is that learning (and retention) is just not possible. It is difficult to conceive of a set of mechanisms that can be so finely tuned to allow for it. Nevertheless, in spite of such evidence against it, learning sometimes does occur. Although the findings of Zovkic and colleagues are novel and important, they also serve to emphasize how little we understand about this fundamental process.

Sheena Josselyn ${ }^{1,2,3,4}$, Paul W Frankland ${ }^{1,2,3,4}$
${ }^{1}$ Program in Neurosciences and Mental Health, The Hospital for Sick Children, Toronto, Ontario M5G 1 X8, Canada; ${ }^{2}$ Institute of Medical Science, University of Toronto, Toronto, Ontario M5S 1A8, Canada; ${ }^{3}$ Department of Psychology, University of Toronto, Toronto, Ontario M5S 3GM, Canada; ${ }^{4}$ Department of Physiology, University of Toronto, Toronto, Ontario M5S 1A8, Canada

Correspondence: Paul W Frankland

E-mail: paul.frankland@sickkids.ca

\section{References}

1 Agranoff BW, Davis RE, Casola L, et al. Science 1967; 158:1600-1601.

2 Vetere G, Restivo L, Cole CJ, et al. Proc Natl Acad Sci USA 2011; 108:8456-8460.

3 Cole CJ, Mercaldo V, Restivo L, et al. Nat Neurosci 2012; 15:1255-1264

4 Josselyn SA. J Psychiatry Neurosci 2010; 35:221-228.

5 Miller CA, Campbell SL, Sweatt JD. Neurobiol Learn Mem 2008; 89:599-603.

6 Peixoto L, Abel T. Neuropsychopharmacology 2013; 38:62-76.

7 Iizuka M, Smith MM. Curr Opin Genet Dev 2003; 13:154-160.

8 Luger K. Curr Opin Genet Dev 2003; 13: 127-135.

9 Zovkic IB, Paulukaitis BS, Day JJ, et al. Nature 2014; 515:582-586.

10 Frankland PW, Bontempi B. Nat Rev Neurosci 2005; 6:119-130.

11 Lashley KS. In Society of Experimental Biology Symposium, No. 4: Psychological mechanisms in animal behavior. Cambridge University Press, 1950. 454-480. 